\title{
Extended Reality for Chronic Pain Relief
}

\author{
Jiaheng Wang \\ Victoria University of Wellington \\ Wellington, New Zealand \\ jiaheng.wang@ecs.vuw.ac.nz \\ Brian Robinson \\ Victoria University of Wellington \\ Wellington, New Zealand \\ brian.robinson@vuw.ac.nz
}

\author{
Craig Anslow \\ Victoria University of Wellington \\ Wellington, New Zealand \\ craig.anslow@ecs.vuw.ac.nz \\ Simon McCallum \\ Victoria University of Wellington \\ Wellington, New Zealand \\ simon.mccallum@ecs.vuw.ac.nz
}

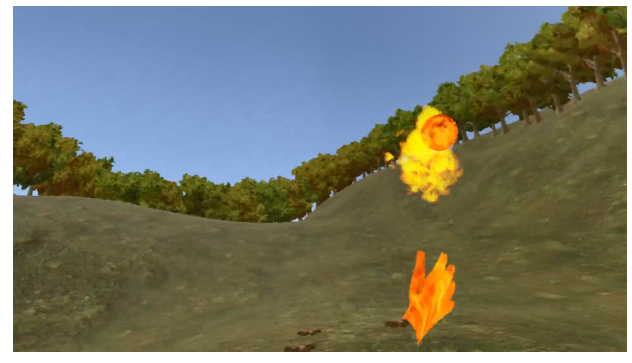

(a) Throwing a fireball to light campfires

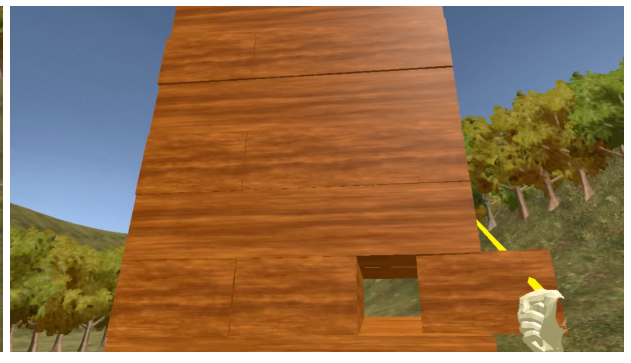

(b) Pulling a block out of a tower

Figure 1: Screenshots of the prototype in action.

\begin{abstract}
Chronic pain is ongoing pain lasting for long periods of time after the initial injury or disease has healed. Chronic pain is difficult to treat and can affect the daily lives of patients. Distraction therapy is a proven way of relieving pain by redirecting the focus of patients' attention. Virtual reality is an effective platform for distraction therapy as it immerses the user visually, aurally, and even somewhat physically in a virtual world detached from reality. There is little research done on the effects that physical interactions have on pain management. This project aims to evaluate different types of extended reality (XR) interactions, including full body movement, for chronic pain patients to determine which is the best for pain relief. We are building a prototype for participants to interact both mentally and physically and measuring the reduction in subjective pain ratings at various points of the XR experience.
\end{abstract}

\section{CCS CONCEPTS}

- Human-centered computing $\rightarrow$ Mixed / augmented reality; Virtual reality; Interaction techniques; Empirical studies in HCI; Activity centered design; • Applied computing $\rightarrow$ Consumer health.

Permission to make digital or hard copies of part or all of this work for personal or classroom use is granted without fee provided that copies are not made or distributed for profit or commercial advantage and that copies bear this notice and the full citation on the first page. Copyrights for third-party components of this work must be honored

For all other uses, contact the owner/author(s).

VRST '19, November 12-15, 2019, Parramatta, NSW, Australia

(c) 2019 Copyright held by the owner/author(s).

ACM ISBN 978-1-4503-7001-1/19/11.

https://doi.org/10.1145/3359996.3365030

\section{KEYWORDS}

virtual reality, augmented reality, mixed reality, chronic pain, pain relief, pain management, user study

\section{ACM Reference Format:}

Jiaheng Wang, Craig Anslow, Brian Robinson, and Simon McCallum. 2019. Extended Reality for Chronic Pain Relief. In 25th ACM Symposium on Virtual Reality Software and Technology (VRST '19), November 12-15, 2019, Parramatta, NSW, Australia. ACM, New York, NY, USA, 2 pages. https: //doi.org/10.1145/3359996.3365030

\section{INTRODUCTION}

Pain is commonly divided into two categories: acute and chronic [Taub et al. 1998]. Acute pain is caused by disease, injury, or some other form of stimulation [Taub et al. 1998], and serves as a biological alert to prevent further damage [Moriarty and Finn 2014]. Chronic pain is an ongoing pain often defined by the length of time [Grichnik and Ferrante 1991]. Chronic pain is more difficult to treat as, by definition, it does not respond to the treatment which resolved the initial source of acute pain. The persistence of chronic pain also has an effect on patients' everyday life such as loss of sleep or unable to perform basic self-care tasks.

Current treatment procedures combine medication with rehabilitation and education. Various forms of analgesia each have their own side-effects. Distraction therapy is a non-pharmacological treatment for pain by directing the attention of the patient to a pleasant event or stimulation to distract the patient from the pain [Fernandez 1986].

As VR headsets become more widespread, what was once obscure uses for such devices gradually becomes mainstream. By engaging 
multiple sensory systems, it is capable of taking users out of their real-world environment and placing them in any virtual world of their choosing, fully immersing them in that environment and filling the user's cognition with the pleasant stimulants for distraction therapy.

While there have been many studies on the effects of VR on subjective pain in a range of settings including burn patients [Hoffman et al. 2000], labour [Frey et al. 2018], surgery [Chan and Scharf 2017], cancer [Mohammad and Ahmad 2018], and dentistry [Wiederhold et al. 2014], most of these studies were unable to engage the full body movement aspects of XR as they were used for acute pain during medical procedures. It would be extremely dangerous for patients to be walking around the room waving their arms around while medical staff are trying to work on them. So far there have been very little research into the differences between physical and mental demand for pain management. This project proposes to develop a variety of interactive content to be delivered through VR and AR and evaluate the effect on chronic pain sufferers to determine which elements of the experience provides the most effective relief to patients.

[Bianchi-Berthouze et al. 2007] previously conducted user studies on full-body interactions and its contributions to the experience. They found that full-body movements not only increased players' engagement but also presence. [Hoffman et al. 2004] conducted many studies into how a sense of presence affected the analgesic effects of the experience and found that stronger presence, from both software and hardware, led to a decrease in pain ratings. Based on this, we hypothesize that by physically engaging the patients, the effects on pain ratings will be better than with mental engagement.

\section{CHRONIC XR}

We are designing and developing a prototype to be used as a platform to conduct user studies. The prototype is designed to have distinctly physical activities and distinctly mental activities, in order to better understand the analgesic effects of each. A passive observation component is also included to act as a control group for the user study. The content is kept the same between VR and AR, with the only difference being the surrounding environment VR has a virtual, made-up environment whereas AR's environment is the real world.

Physical activities engage users by requiring either fine motor skills or hand-eye coordination. Although full body interactions are implemented, they are not required to fully engage with the experience. Full body interaction includes walking around the play area and interacting with the world by moving arms and hands, utilizing both upper and lower limbs. But with the target demographics being chronic pain patients, accessibility features are provided to reduce strain or excessive movement on pain regions. This includes functions like teleporting (Figure 2) instead of walking and minimizing the angles users have to move their arms. For the same reason, activities requiring vigorous movement or fast reflexes will not be included.

Mental activities engage users by requiring memorization, simple arithmetic, or logical thinking. Although these activities still require physical movement to interact, the challenge and cognitive demand will not be on the physical side.

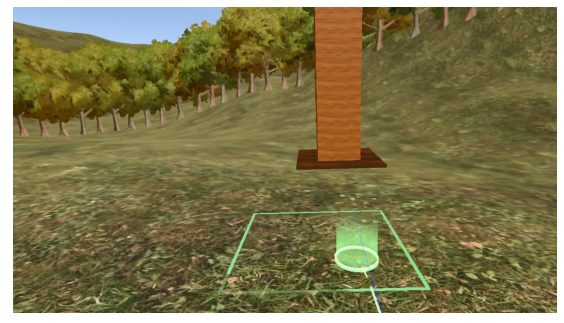

Figure 2: Teleport system

The virtual activities have a magical theme. Users can use various spells such as telekinesis, teleportation, and controlling elements (fire, water, lightning, etc.) Users interact with the world via controllers, using the touchpad to select the spell they want, and pulling the trigger to activate the spell. There is also a no-spell mode where users can pick up and throw objects around without magic.

The game is comprised of a series of "rooms" or levels. The player completes each level by getting a key and placing the key into the keyhole. The challenges lie in obtaining the key.

We presented a prototype of an XR game which forms the beginning of a research project to better examine using XR for chronic pain relief. A user study with chronic pain patients will be conducted to evaluate the analgesic effects. We hope the results of this study can influence the design of future pain management games to be more effective and help pain patients have a better quality of life.

\section{REFERENCES}

Nadia Bianchi-Berthouze, Whan Woong Kim, and Darshak Patel. 2007. Does body movement engage you more in digital game play? and why?. In International conference on affective computing and intelligent interaction. Springer, 102-113.

Peter Y Chan and Simon Scharf. 2017. Virtual reality as an adjunctive nonpharmacological sedative during orthopedic surgery under regional anesthesia: A pilot and feasibility study. Anesthesia \& Analgesia 125, 4 (2017), 1200-1202.

Ephrem Fernandez. 1986. A classification system of cognitive coping strategies for pain. Pain 26, 2 (1986), 141-151.

David P Frey, Melissa E Bauer, Carrie L Bell, Lisa Kane Low, Afton L Hassett, Ruth B Cassidy, Katherine D Boyer, and Sam R Sharar. 2018. Virtual Reality Analgesia in Labor: The VRAIL Pilot Study-A Preliminary Randomized Controlled Trial Suggesting Benefit of Immersive Virtual Reality Analgesia in Unmedicated Laboring Women. Anesthesia and analgesia (2018).

KP Grichnik and FM Ferrante. 1991. The difference between acute and chronic pain. The Mount Sinai journal of medicine, New York 58, 3 (1991), 217-220.

Hunter G Hoffman, Jason N Doctor, David R Patterson, Gretchen J Carrougher, and Thomas A Furness III. 2000. Virtual reality as an adjunctive pain control during burn wound care in adolescent patients. Pain 85, 1-2 (2000), 305-309.

Hunter G Hoffman, Sam R Sharar, Barbara Coda, John J Everett, Marcia Ciol, Todd Richards, and David R Patterson. 2004. Manipulating presence influences the magnitude of virtual reality analgesia. Pain 111, 1-2 (2004), 162-168.

Eslam Bani Mohammad and Muayyad Ahmad. 2018. Virtual reality as a distraction technique for pain and anxiety among patients with breast cancer: A randomized control trial. Palliative \& supportive care (2018), 1-6.

Orla Moriarty and David P Finn. 2014. Cognition and pain. Current opinion in supportive and palliative care 8,2 (2014), 130-136.

Neal S Taub, Gregory M Worsowicz, Steve M Gnatz, and David X Cifu. 1998. 1. Definitions and diagnosis of pain. Archives of physical medicine and rehabilitation 79, 3 (1998), S49-S53.

Mark D Wiederhold, Kenneth Gao, and Brenda K Wiederhold. 2014. Clinical use of virtual reality distraction system to reduce anxiety and pain in dental procedures. Cyberpsychology, Behavior, and Social Networking 17, 6 (2014), 359-365. 